PROCEEDINGS OF THE

AMERICAN MATHEMATICAL SOCIETY

Volume 139, Number 9, September 2011, Pages 3191-3193

S 0002-9939(2011)11026-8

Article electronically published on March 24, 2011

\title{
FAITHFUL REPRESENTATIONS OF ASSOCIATION SCHEMES
}

\author{
AKIHIDE HANAKI \\ (Communicated by Jim Haglund)
}

\begin{abstract}
Every character of an association scheme can be considered as a faithful character of some quotient scheme. Also we will show that a faithful character of an association scheme determines a thin closed subset which is cyclic as a finite group.
\end{abstract}

\section{INTRODUCTION}

Let $G$ be a finite group and let $\chi$ be a character of $G$. We can consider that $\chi$ is a faithful character of $G / \operatorname{Ker}(\chi)$. If $G$ has a faithful irreducible character, then the center of $G$ is cyclic. These are well known facts in group representation theory. We will generalize them to characters of association schemes.

Every character of an association scheme can be considered as a faithful character of some quotient scheme (Theorem 2.1). Also we will show that a faithful character of an association scheme determines a thin closed subset which is cyclic as a finite group (Theorem 3.1).

Let $(X, S)$ be an association scheme in the sense of [7] or [3]. We will denote the valency of $s \in S$ by $n_{s}$. Let $T$ be a closed subset of $S$. Put $e_{T}=n_{T}^{-1} \sum_{t \in T} \sigma_{t}$. Then $e_{T}$ is an idempotent of $\mathbb{C} S$. It is known that $\mathbb{C}(S / / T) \cong e_{T} \mathbb{C} S e_{T}$ as algebras by $\sigma_{s^{T}} \mapsto\left(n_{s^{T}} / n_{s}\right) e_{T} \sigma_{s} e_{T}$ (see [4).

We denote the identity matrix by $E$.

\section{FAITHFUl REPRESENTATIONS}

Let $(X, S)$ be an association scheme, and let $\Phi: \mathbb{C} S \rightarrow M_{n}(\mathbb{C})$ be a representation of $(X, S)$ affording a character $\varphi$. Define

$$
K(\Phi)=\left\{s \in S \mid \Phi\left(\sigma_{s}\right)=n_{s} E\right\}
$$

and

$$
K(\varphi)=\left\{s \in S \mid \varphi\left(\sigma_{s}\right)=n_{s} \varphi(1)\right\} .
$$

It is known that $K(\Phi)=K(\varphi)$ (see [1, section 3]). Note that $K(\Phi)$ is closed but not necessarily normal. We say that $\Phi$ or $\varphi$ is faithful if $K(\Phi)=\{1\}$.

If $K(\Phi)$ is a normal closed subset of $S$, then there is a natural algebra epimorphism $\pi: \mathbb{C} S \rightarrow \mathbb{C}(S / / K(\Phi))$ and $\Phi$ can be considered as a representation of $S / / K(\Phi)$. But $K(\Phi)$ is not necessarily normal, and the natural map $\pi: \mathbb{C} S \rightarrow$ $\mathbb{C}(S / / K(\Phi))$ is not an algebra homomorphism in general (see [6]).

Received by the editors August 19, 2010.

2010 Mathematics Subject Classification. Primary 05E30.

Key words and phrases. Association scheme, representation, character, faithful.

(C)2011 American Mathematical Society Reverts to public domain 28 years from publication 319 
The next theorem is the main result in this section.

Theorem 2.1. Let $(X, S)$ be an association scheme, and let $\Phi: \mathbb{C} S \rightarrow M_{n}(\mathbb{C})$ be a representation of $(X, S)$. Suppose that $T$ is a closed subset contained in $K(\Phi)$. Then we can define a representation $\Phi^{\prime}: \mathbb{C}(S / / T) \rightarrow M_{n}(\mathbb{C})$ by $\Phi^{\prime}\left(\sigma_{s^{T}}\right)=\left(n_{s^{T}} / n_{s}\right) \Phi\left(\sigma_{s}\right)$. Moreover, $\Phi^{\prime}$ is faithful if $T=K(\Phi)$.

Proof. If $\Psi$ is an irreducible component of $\Phi$, then $K(\Phi) \subseteq K(\Psi)$. Thus, without loss of generality, we may suppose that $\Phi$ is irreducible.

Let $\varphi$ be the character afforded by $\Phi$. Let $e_{\varphi}$ be the primitive central idempotent of $\mathbb{C} S$ corresponding to $\varphi$. By the assumption on $T$, we have $e_{\varphi} e_{T}=e_{\varphi}=e_{T} e_{\varphi}$.

We will show that $\Phi^{\prime}$ is well-defined. Suppose $s^{T}=u^{T}$. We have

$$
\begin{aligned}
\frac{1}{n_{s}} \Phi\left(\sigma_{s}\right) & =\Phi\left(e_{\varphi} \frac{1}{n_{s}} \sigma_{s} e_{\varphi}\right)=\Phi\left(e_{\varphi} e_{T} \frac{1}{n_{s}} \sigma_{s} e_{T} e_{\varphi}\right) \\
& =\Phi\left(e_{\varphi} e_{T} \frac{1}{n_{u}} \sigma_{u} e_{T} e_{\varphi}\right)=\Phi\left(e_{\varphi} \frac{1}{n_{u}} \sigma_{u} e_{\varphi}\right)=\frac{1}{n_{u}} \Phi\left(\sigma_{u}\right) .
\end{aligned}
$$

This means that $\Phi^{\prime}$ is well-defined.

We show that $\Phi^{\prime}$ is an algebra homomorphism. We use the isomorphism $\mathbb{C}(S / / T)$ $\cong e_{T} \mathbb{C} S e_{T}$ and identify them. Then $\Phi^{\prime}\left(e_{T} \sigma_{s} e_{T}\right)=\Phi\left(\sigma_{s}\right)$. We have

$$
\begin{aligned}
\Phi^{\prime}\left(\left(e_{T} \sigma_{s} e_{T}\right)\left(e_{T} \sigma_{u} e_{T}\right)\right) & =\Phi\left(\sigma_{s} e_{T} \sigma_{u}\right)=\Phi\left(\sigma_{s} e_{T}\right) \Phi\left(\sigma_{u}\right) \\
& =\Phi\left(\sigma_{s} e_{T} e_{\varphi}\right) \Phi\left(\sigma_{u}\right)=\Phi\left(\sigma_{s} e_{\varphi}\right) \Phi\left(\sigma_{u}\right) \\
& =\Phi\left(\sigma_{s}\right) \Phi\left(\sigma_{u}\right)=\Phi^{\prime}\left(e_{T} \sigma_{s} e_{T}\right) \Phi^{\prime}\left(e_{T} \sigma_{u} e_{T}\right) .
\end{aligned}
$$

Finally, we will show that $\Phi^{\prime}$ is faithful if $T=K(\Phi)$. Suppose $s^{T} \in K\left(\Phi^{\prime}\right)$. Then $E=n_{s^{T}}{ }^{-1} \Phi^{\prime}\left(\sigma_{s^{T}}\right)=n_{s}{ }^{-1} \Phi\left(\sigma_{s}\right)$. So $s \in K(\Phi)$ and $s^{T}=1^{T}$. Now $\Phi^{\prime}$ is faithful.

Corollary 2.2. Let $(X, S)$ be an association scheme, and let $\varphi$ be a character of $(X, S)$. Suppose that $T$ is a closed subset contained in $K(\varphi)$. Then $n_{u}{ }^{-1} \varphi\left(\sigma_{u}\right)=$ $n_{s}{ }^{-1} \varphi\left(\sigma_{s}\right)$ for any $u \in T s T$.

Proof. This is obtained by the fact that $\Phi^{\prime}$ in Theorem 2.1 is well-defined.

\section{Faithful Representations And Closed subsets}

Let $(X, S)$ be an association scheme, and let $\Phi: \mathbb{C} S \rightarrow M_{n}(\mathbb{C})$ be a representation of $(X, S)$ affording a character $\varphi$. Define

$$
Z(\varphi)=\left\{s \in S|| \varphi\left(\sigma_{s}\right) \mid=n_{s} \varphi(1)\right\}
$$

Then $Z(\varphi)$ is a closed subset of $S$ containing $K(\varphi)$ (see [2, Proposition 3.2 and 3.3]). For $s \in S, s \in Z(\varphi)$ if and only if $\Phi\left(\sigma_{s}\right)=\varepsilon_{s} n_{s} E$ for some root of unity $\varepsilon_{s}$.

The following theorem is a generalization of [5, Theorem 2.32 (a)].

Theorem 3.1. Let $\varphi$ be a faithful character of $(X, S)$. Then $Z(\varphi)$ is thin and cyclic as a finite group.

In the rest of this section, $(X, S)$ is an association scheme and $\Phi: \mathbb{C} S \rightarrow M_{n}(\mathbb{C})$ is a faithful representation of $(X, S)$ affording a character $\varphi$. For $u \in Z(\varphi)$ we define a root of unity $\varepsilon_{u}$ by $\Phi\left(\sigma_{u}\right)=\varepsilon_{u} n_{u} E$ or equivalently by $\varphi\left(\sigma_{u}\right)=\varepsilon_{u} n_{u} \varphi(1)$. We need a lemma.

Lemma 3.2. If $u, v \in Z(\varphi)$ and $u \neq v$, then $\varepsilon_{u} \neq \varepsilon_{v}$. Moreover, $Z(\varphi)$ is thin. 
Proof. For $u, v \in Z(\varphi)$, suppose $\varepsilon_{u}=\varepsilon_{v}$. Then $\Phi\left(\sigma_{u}\right) \Phi\left(\sigma_{v^{*}}\right)=n_{u} n_{v^{*}} E$. Now $w \in K(\varphi)=\{1\}$ for any $w \in u v^{*}$. So $u v^{*}=\{1\}$. This means that $u=v$ and $u$ is thin.

Proof of Theorem 3.1. If $\xi$ is an irreducible constituent of $\varphi$, then $Z(\varphi) \subseteq Z(\xi)$. So we may suppose that $\varphi$ is irreducible. We consider $Z(\varphi)$ as a finite group. Then, by Lemma 3.2, $\sigma_{u} \mapsto \varepsilon_{u}$ is an irreducible faithful character of an abelian group $Z(\varphi)$. So $Z(\varphi)$ is cyclic.

We remark that if $\varphi$ is a faithful irreducible character of a finite group $G$, then $Z(\varphi)$ is just the center of $G$. But for a character of an association scheme, it is not true in general.

\section{ACKNOWLEDGEMENT}

The author would like to thank the referee for many helpful comments.

\section{REFERENCES}

1. A. Hanaki, Characters of association schemes and normal closed subsets, Graphs Combin. 19 (2003), no. 3, 363-369. MR2007898 (2004h:05132)

2. _ Nilpotent schemes and group-like schemes, J. Combin. Theory Ser. A 115 (2008), no. 2, 226-236. MR2382513 (2008m:05314)

3. $ـ$ Representations of finite association schemes, European J. Combin. 30 (2009), no. 6 , 1477-1496. MR2535397 (2010g:05395)

4. A. Hanaki and M. Hirasaka, Theory of Hecke algebras to association schemes, SUT J. Math. 38 (2002), no. 1, 61-66. MR1919947(2003g:05134)

5. I. M. Isaacs, Character theory of finite groups, Academic Press [Harcourt Brace Jovanovich Publishers], New York, 1976. MR0460423 (57:417)

6. B. Xu, On morphisms of association schemes, J. Algebra 322 (2009), no. 4, 1013-1028. MR.2537669 (2010i:05358)

7. P.-H. Zieschang, An algebraic approach to association schemes, Lecture Notes in Mathematics, vol. 1628, Springer-Verlag, Berlin, 1996. MR1439253 (98h:05185)

Faculty of Science, Shinshu University, Matsumoto 390-8621, Japan

E-mail address: hanaki@shinshu-u.ac.jp 